\title{
Vibrationally resolved photoelectron-spin-polarization spectroscopy of HI molecules
}

\author{
N. Böwering, M. Salzmann, M. Müller, ${ }^{*}$ H.-W. Klausing, and U. Heinzmann \\ Fakultät für Physik, Unicersität Bielefeld, D-4800 Bielefeld, Germany \\ and Fritz-Haber-Institut der Max-Planck-Gesellschaft, D-1000 Berlin 33, Germany
}

(Received 4 September 1991)

\begin{abstract}
The photoemission from $\mathrm{HI}$ is studied separately for different vibrational levels of the ${ }^{2} \Pi$ final ionic states using spin-resolved photoelectron spectroscopy with circularly polarized light. Constant-ionicstate measurements of the cross sections $\sigma$ and the polarization parameters $A$ indicate that all channels are strongly influenced by autoionization resonances. By combining these data, sums of partial contributions to $\sigma$ are analyzed in detail to obtain specific information on the coupling of the resonances to the outgoing partial waves $\epsilon \lambda$. Apart from generally similar trends, the results show pronounced variations for different vibrational levels.
\end{abstract}

PACS number(s): $33.20 . \mathrm{Ni}, 33.60 . \mathrm{Cv}, 33.80 . \mathrm{Eh}, 33.10 . \mathrm{Gx}$

Molecular photoionization dynamics can be probed by studies of the photoelectron-spin polarization. To that end, experimental and theoretical methods were developed in recent years. Energy- and angle-integrated measurements of the polarization parameter $A$ were carried out with circularly polarized synchrotron radiation [1]. The general nonrelativistic theory of Cherepkov [2] was applied in the first $a b$ initio calculations of the polarization parameters to the spin-orbit ionization region of $\mathrm{HI}$ [3] and to the open continuum for $\mathrm{HI}$ and $\mathrm{HBr}$ [4]. As compared to the atomic case, however, molecular photoionization is more complicated due to molecular vibration and rotation, and methods directed at analyzing related polarization structures are beginning to emerge. A theoretical treatment including rotation explicitly in both initial and final states was given by Raseev and Cherepkov [5] and applied to $\mathrm{HI}$ in Ref. [6]. By resolving the rotational spacing in the excitation step, the angle-integrated photoelectron polarization was examined recently for the spinorbit autoionization region of $\mathrm{HI}$ [7]. Here, we report on polarization measurements where the molecular vibration has been resolved for the final ionic states.

Owing to their related nature it can be expected that the spin-polarization parameters should exhibit a dependence on vibrational excitation in a similar fashion as the spin-independent dynamical photoionization parameters $\sigma$ (partial cross section) and $\beta$ (angular distribution parameter). In general, only if special features are present in the photoionization spectra (like autoionization or shape resonances) have pronounced variations been observed for the different vibrational levels of a final ionic state with respect to the cross section branching ratios and the asymmetry parameters $\beta$ (for a review, see Ref. [8]). In particular, Carlson has first reported a strong variation of $\beta$ in the ionization of $\mathrm{N}_{2}$ and $\mathrm{O}_{2}$ due to the influence of autoionization [9]. For HI, Carlson et al. [10] have also observed a more isotropic dependence for $\beta$ for $v=1$ as compared to $v=0$ for the $\mathrm{HI}^{+} X^{2} \Pi_{3 / 2}$ final ionic state. On the other hand, for $\mathrm{H}_{2}$ no strong $v$ 'dependence was found in a wide energy range as reported recently [11]. Likewise, as was also noted before [2], a vibrational dependence might occur for the degree of photoelectron-spin po- larization. To examine this, the $5 p \pi$ ionization of HI was chosen, since the spin-orbit splitting of $\mathrm{HI}^{+}{ }^{2} \Pi$ is large and significant population of vibrationally excited states occurs in the range of $11-13-\mathrm{eV}$ photon energy [12].

The angle-, energy-, and spin-resolved experiment was carried out with circularly polarized synchrotron radiation emitted out of plane at the storage ring BESSY using the apparatus described in detail previously [13]. The photoelectrons emitted at the magic angles are analyzed with a hemispherical spectrometer and the spin-polarization parameter $\boldsymbol{A}$ [characterizing the spin-polarization component $A(\theta)$ parallel to the photon momentum] is determined using a subsequent Mott detector. Constant-ionicstate intensity data were recorded at a spectrometer resolution of $\Delta E \approx 80 \mathrm{meV}$ and an optical resolution of $\Delta \lambda=0.17 \mathrm{~nm}$ while spin-polarization measurements were performed at $\Delta E \approx 180 \mathrm{meV}$ and $\Delta \lambda=0.5 \mathrm{~nm}$ for intensity reasons.

A representative photoelectron spectrum with vibrational excitation of $\mathrm{HI}^{+} X^{2} \Pi_{i}$ is shown in Fig. 1. It is in contrast to the HeI spectrum [14] which reflects direct ionization only and does not show levels with $v>1$. The "non-Franck-Condon" population of vibrationally excited

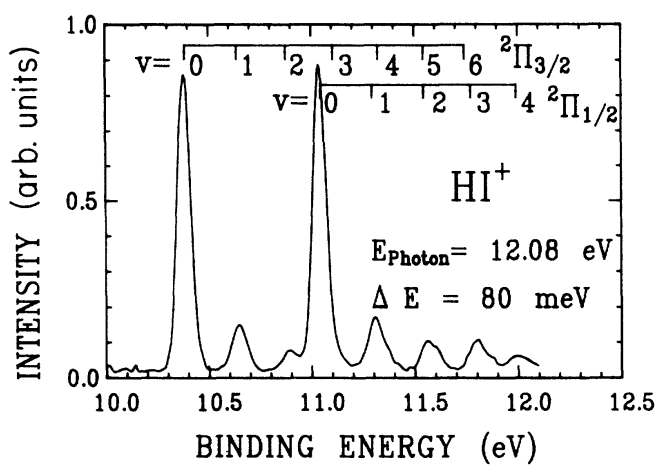

FIG. I. Vibrationally resolved photoelectron spectrum for $\mathrm{HI}^{+} X^{2} \Pi$ final ionic states. The data (typical relative errors of $10 \%)$ are corrected for the spectrometer transmission characteristics. 
levels arises due to the influence of electronic autoionization resonances converging to the $\mathrm{HI}^{+} A^{2} \Sigma_{1 / 2}^{+}$state as discussed in detail elsewhere [12]. In Fig. 2 the partial photoionization cross sections $\sigma$ obtained for the levels $v=0$ and $v=1$ are displayed. The absolute cross section scale ( $\sim 20 \%$ error) was established by matching our data to those of Carlson et al. [15] at higher photon energies as described elsewhere [16].

The data of Fig. 2 exhibit a pronounced structure due to autoionization resonances. Two different regions can be distinguished for each vibrational level: Region I occurring between the two ${ }^{2} \Pi$ ionization limits is dominated by spin-orbit autoionization of Rydberg states converging to the ${ }^{2} \Pi_{1 / 2} v=0$ and $v=1$ limits, respectively. Region II above the ${ }^{2} \Pi_{1 / 2}$ limits is mainly influenced by electronic autoionization. Here, striking differences are observed for the $v=0$ and $v=1$ levels, whereas the two spin-orbit components each show similar structures. The results for region I are in agreement with those of Carlson et al. [10] obtained at slightly higher resolution. Only the coarse resonance structures due to the series with $(5 p \pi)^{32} \Pi_{1 / 2}$ core and $n d \pi, \delta$ Rydberg electron orbitals are visible in contrast to the high-resolution total yield results obtained with frequency-converted laser radiation [17,18]. However, here the vibrational levels are separated and the analogies in the structures for the ${ }^{2} \Pi_{3 / 2} v=0$ and $v=1$ channels become apparent when the energy scale is shifted by the vibrational excitation energy for the $v=1$ channel. The correspondence of the coarse intensity variations indicates that the preeminent spin-orbit autoionization processes take place for $v=1$ state in a similar fashion as for the $v=0$ state (i.e., $\Delta v=0$ seems to dominate, see also data of Ref. [10]). For region II partial cross sections have not been reported before. A broad resonance structure centered near $12.4 \mathrm{eV}$ is observed for the intense $v=0$ channels, in agreement with total yield results $[19,20]$. The
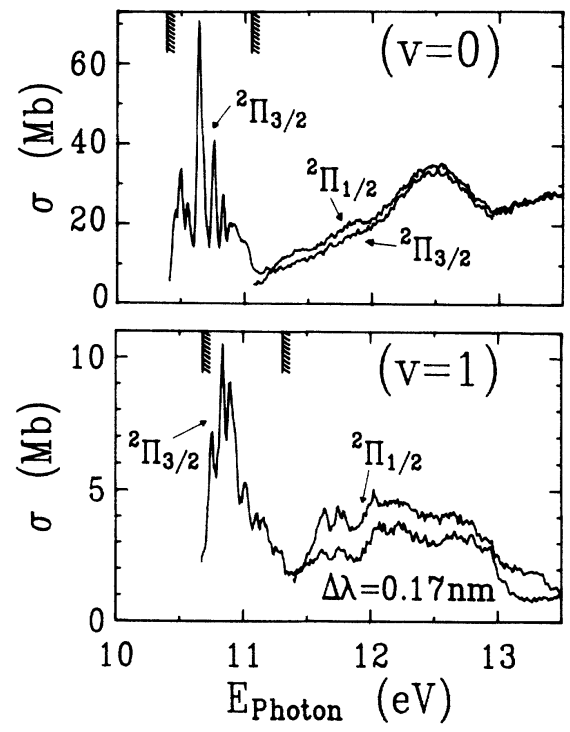

FIG. 2. Partial photoionization cross sections for $\mathrm{HI}^{+}$ $X^{2} \Pi_{i} v=0,1$ final ionic states. Ionization limits are indicated by vertical lines. $v=1$ states also exhibit broad features; in addition, several narrower resonances are superimposed in the energy region of $11.6-12.1 \mathrm{eV}$. The broadened nature of the resonances can be attributed to strong predissociation of the Rydberg states with $A^{2} \Sigma^{+}$core $[12,19,20]$. From the effective quantum number and in analogy to the lighter hydrogen halides $[19,21]$, the broad resonance in the $v=0$ channels is assigned to $d \pi$ Rydberg states, whereas the resonance structure near $11.8 \mathrm{eV}$ could be due to $p \pi \mathrm{Ryd}$ berg states. The cross sections indicate that the coupling via the resonances must be considerably different for $v=0$ and $v=1$ final states, as was also found in the corresponding autoionization structures for $\mathrm{HCl}$ [22].

The spin-polarization results obtained for the $v=0$ and $v=1$ final ionic states are summarized in Fig. 3. We concentrated on the final ionic states $\mathrm{HI}^{+}{ }^{2} \Pi_{3 / 2} v=0$, ${ }^{2} \Pi_{3 / 2} v=1$, and ${ }^{2} \Pi_{1 / 2} v=0$, since these states could be separated unambiguously (see Fig. 1). For $\mathrm{HI}^{+}{ }^{2} \Pi_{1 / 2}$ states, small admixtures from vibrationally excited levels of $\mathrm{HI}^{+}{ }^{2} \Pi_{3 / 2}$ cannot be ruled out completely, although background polarization contributions to the polarization were always subtracted by measurements at energies above and below each photoelectron peak. Measurements for vibrationally excited states required typically $2-4 \mathrm{~h}$ of data accumulation per data point.

In Fig. 3, generally the so-called nonrelativistic relationship of equal magnitude but opposite sign for the $A$ parameter for the two spin-orbit states [2] is fulfilled remarkably well, except for the energy region of 11.3-11.6 eV. In region $I$, the polarization values are smaller by about a factor of 2 for ${ }^{2} \Pi_{3 / 2} v=1$ as compared to ${ }^{2} \Pi_{3 / 2} v=0$ (the values for the parameter $\beta$ are also smaller [10]), whereas for region II of electronic autoionization this trend is reversed. In contrast to the fairly weak variations observed for $\sigma$ (Fig. 2) the structures found for $A$ are quite pronounced. The polarization values decrease to almost zero for all ionization channels at $11.7-12.0 \mathrm{eV}$. For $\mathrm{HBr}{ }^{2} \Pi_{i} v=0$ [21] a similar drop to small polariza-

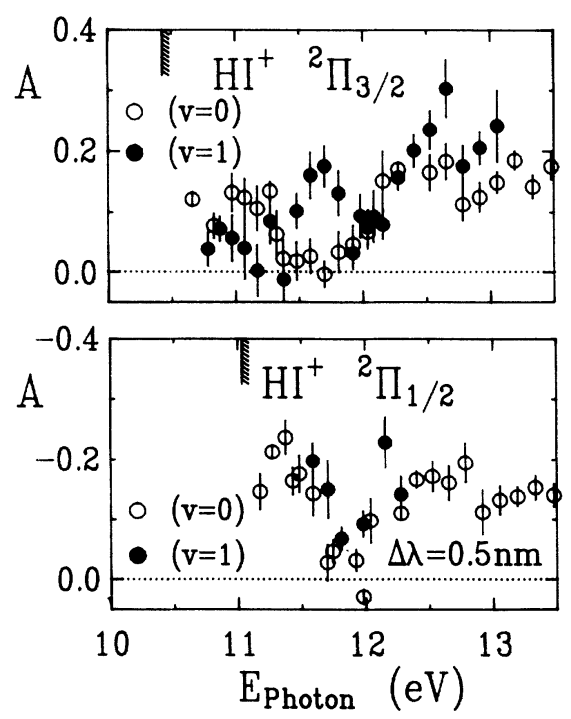

FIG. 3. Photon energy dependence for the polarization parameters $A$ for $\mathrm{HI}^{+}$final ionic states. 
tion values occurs in the region between the $d \pi$ and $p \pi$ autoionization resonances. When comparing the polarization data for the ${ }^{2} \Pi_{3 / 2} v=0$ and $v=1$ channels, the most striking difference occurs at around $11.6 \mathrm{eV}$, whereas in the energy region from $11.9-12.4 \mathrm{eV}$ the values are similar.
For further data analysis it is instructive to consider the outgoing partial waves $\epsilon l \lambda$ involved and to discuss the results in terms of partial contributions with respect to $\lambda$. $(\lambda$ is the projection of the electron orbital angular momentum $l$ on the molecular axis.) The electronic autoionization process of interest here is described by

$$
\begin{aligned}
\mathrm{HI}(5 s \sigma)^{2}(5 p \sigma)^{2}(5 p \pi)^{4}{ }^{1} \Sigma_{0}^{+}+h v & \rightarrow \mathrm{HI}^{*}(5 s \sigma)^{2}(5 p \sigma)(5 p \pi)^{4}(n l \sigma)^{1} \Sigma^{+},(n l \pi){ }^{1} \Pi \\
& \rightarrow \mathrm{HI}^{+}(5 s \sigma)^{2}(5 p \sigma)^{2}(5 p \pi)^{3}{ }^{2} \Pi_{3 / 2},{ }^{2} \Pi_{1 / 2}+e^{-}(\epsilon l \sigma, \epsilon l \pi, \epsilon l \delta),
\end{aligned}
$$

with ${ }^{\prime} \Pi$ resonant states yielding only $\epsilon l \sigma$ and $\epsilon l \delta$ partial waves while ' $\Sigma^{+}$resonances give rise only to outgoing $\epsilon l \pi$ waves. The dominant contributions should come from $d$, $p$, and $s$ waves. The resonant states $\mathrm{HI}^{*}$ are subject to predissociation due to crossings with repulsive states with a ${ }^{4} \Pi$ core in analogy to the predissociation of the $A^{2} \Sigma^{+}$ ionic state [12]. In studies of this process for $\mathrm{HCl}$ [23] and $\mathrm{HBr}[21]$ it was found that for the ${ }^{1} \Pi$ resonances ionization predominates over predissociation, whereas the ${ }^{\prime} \Sigma^{+}$states are more strongly predissociated. Therefore, for $\mathrm{HI}$ where the predissociation is even stronger the resonant cross section should be dominated by ' $\Pi$ states.

$\sigma$ and $A$ can be combined to obtain additional information on the photoionization process. Just like the total cross section $\sigma$, within the nonrelativistic theory [2] [rotationless case, Hund's case (a)] the polarization parameter $A$ does also not contain phase-shift differences of the outgoing partial waves and depends only on the partial cross sections $\sigma_{\sigma}, \sigma_{\pi}$, and $\sigma_{\delta}$. The formulas for $\sigma$ and $A$ of Ref. [4] yield sums and differences of partial waves:

$$
\begin{aligned}
& \sigma(\delta, \pi)=\sigma_{\delta}+\sigma_{\pi} / 2=(0.5 \pm A) \sigma, \\
& \sigma(\sigma, \pi)=\sigma_{\sigma}+\sigma_{\pi} / 2=(0.5 \mp A) \sigma, \\
& \sigma(\delta, \sigma)=\sigma_{\delta}-\sigma_{\sigma}= \pm 2 A \sigma,
\end{aligned}
$$

for a given final state of an ionic doublet, here ${ }^{2} \Pi_{3 / 2}$. (The lower sign refers to ${ }^{2} \Pi_{1 / 2}$ states.) Two of the three partitions above are linearly independent; in each case one partial contribution $\sigma_{\lambda}$ is eliminated.

The fact that $A$ is positive for ${ }^{2} \Pi_{3 / 2}$ states and negative for ${ }^{2} \Pi_{1 / 2}$ states reflects that the $\epsilon \delta$ contributions are larger than the $\epsilon \sigma$ contributions throughout the energy range examined here. In Fig. 4 the partial cross-section contributions of Eqs. (1) and (2) are shown for $\mathrm{HI}^{+}{ }^{2} \Pi_{3 / 2} v=0$ and $v=1$ final ionic states. It is apparent that the $d \pi$ resonance in the $v=0$ channel causes a strong enhancement of the $\sigma_{\delta}$ partial contribution; i.e., it couples mainly to $\epsilon d \delta$ waves (since $l=2$ should be the dominant angular momentum). The resonance is almost absent for $\sigma_{\sigma}$ $+\sigma_{\pi} / 2$. Similar results were obtained for the partial sums for the ${ }^{2} \Pi_{1 / 2} v^{\prime}=0$ channel (not shown). In region $I$ the partial sums are large for both $v=0$ and $v=1$. This could be caused by large contributions from $\epsilon l \pi$ waves. The resonance structures for region II of the ${ }^{2} \Pi_{3 / 2} v=1$ final state become more distinct in Fig. 4 than in the crosssection data of Fig. 2. Since in contrast to the $v=0$ channels the contributions to the vibrationally excited states are almost exclusively caused by autoionization (see also Ref. [12]) and since the resonances should be mainly of ' $\Pi$ symmetry, the $\sigma_{\pi}$ partial cross section should be small compared to $\sigma_{\delta}$ and $\sigma_{\sigma}$ in the region above the ${ }^{2} \Pi_{1 / 2} v=1$ limit. Therefore, in the range from 11.3-13.1 eV Fig. 4 shows essentially only the $\sigma_{\delta}$ and $\sigma_{\sigma}$ partial contributions to ${ }^{2} \Pi_{3 / 2} v=1$. Again, the $\sigma_{\delta}$ contribution dominates and at around 11.7 and $12.6 \mathrm{eV}$ it is more than twice as intense as compared to $\sigma_{\sigma}$ giving rise to relatively large polarization values.

In order to examine possible similarities in the photoionization process for a sequence of vibrational levels for the final states, six different levels were examined at photon energies of 12.28 and $12.16 \mathrm{eV}$. This corresponds to a region where the differences in $A$ between the $v=0$ and $v=1$ channels are relatively small. The results are given in Table I. Since the cross sections differ it is advantageous to compare the fractional contributions to the different partial $\sigma_{\lambda}$ sums in addition to the evolution of the electron polarization. In all cases the $\sigma_{\delta}+\sigma_{\pi} / 2$ contribution is largest and the sign of the polarization is consistent with a dominance of $\sigma_{\delta}$. For the data set at $12.28 \mathrm{eV}$ there are close similarities in the partial $\sigma_{\lambda}$ fractions for the various states, apart perhaps from ${ }^{2} \Pi_{1 / 2} v=2$. However, the variations in the results for the vibrational levels of the set taken at $12.16 \mathrm{eV}$ demonstrate that a close correspondence happens only incidentally. Rather, these

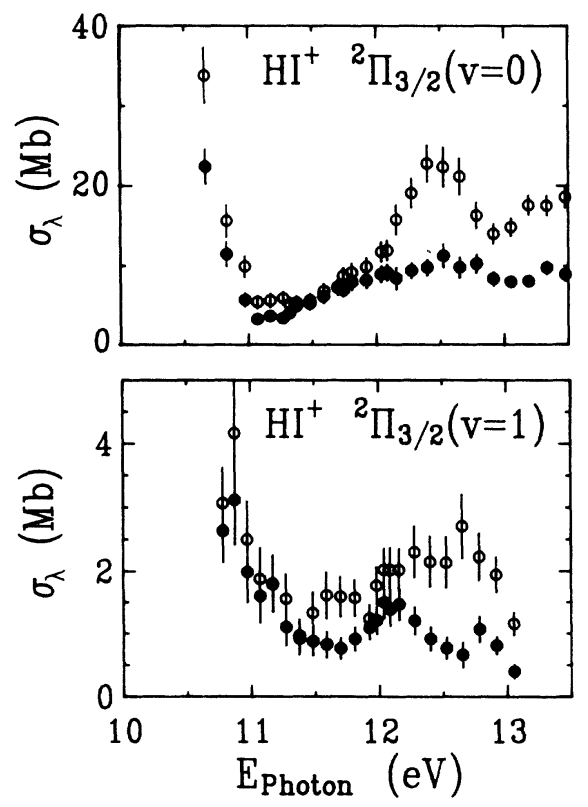

FIG. 4. Energy dependence for sums of partial contributions $\sigma_{\lambda}$ to the cross sections for $\mathrm{HI}^{+} X^{2} \Pi_{3 / 2} v=0$ and $v^{v}=1$ final ionic states (open circles, $\sigma_{\delta}+\sigma_{\pi} / 2$; solid circles, $\sigma_{\sigma}+\sigma_{\pi} / 2$ ). 
TABLE I. Comparison of polarization parameter $A$, cross section $\sigma$, and fractions of $\sigma_{\lambda}$ partial sums for different vibrational levels of the ${ }^{2} \Pi_{3 / 2}$ and ${ }^{2} \Pi_{1 / 2}$ final ionic states at the same photon energy [see also Eqs. (1) and (2)]. $\sigma(\delta, \sigma) / \sigma$ is obtained by forming the difference of the last two columns.

\begin{tabular}{|c|c|c|c|c|}
\hline Final state & $A(\%)$ & $\sigma(\mathrm{Mb})$ & $\sigma(\delta, \pi) / \sigma$ & $\sigma(\sigma, \pi) / \sigma$ \\
\hline \multicolumn{5}{|c|}{ Photon energy: $12.28 \mathrm{eV}(101 \mathrm{~nm})$} \\
\hline${ }^{2} \Pi_{3 / 2} \quad v=0$ & $17.0 \pm 1.1$ & $28.4 \pm 2.7$ & $0.67 \pm 0.01$ & $0.33 \pm 0.01$ \\
\hline${ }^{2} \Pi_{3 / 2} v=1$ & $15.7 \pm 2.0$ & $3.4 \pm 0.6$ & $0.66 \pm 0.02$ & $0.34 \pm 0.02$ \\
\hline${ }^{2} \Pi_{1 / 2} \quad v=0$ & $-11.0 \pm 1.1$ & $29.5 \pm 3.4$ & $0.61 \pm 0.01$ & $0.39 \pm 0.01$ \\
\hline${ }^{2} \Pi_{1 / 2} \quad v=1$ & $-14.2 \pm 3.0$ & $4.3 \pm 0.8$ & $0.64 \pm 0.03$ & $0.36 \pm 0.03$ \\
\hline${ }^{2} \Pi_{1 / 2} \quad v=2$ & $-21.8 \pm 4.0$ & $3.5 \pm 0.7$ & $0.72 \pm 0.04$ & $0.28 \pm 0.04$ \\
\hline${ }^{2} \Pi_{1 / 2} \quad v=3$ & $-12.3 \pm 5.5$ & $3.7 \pm 0.7$ & $0.62 \pm 0.06$ & $0.38 \pm 0.06$ \\
\hline \multicolumn{5}{|c|}{ Photon energy: $12.16 \mathrm{eV}(102 \mathrm{~nm})$} \\
\hline${ }^{2} \Pi_{3 / 2} \quad l=0$ & $15.1 \pm 4.9$ & $24.1 \pm 2.2$ & $0.65 \pm 0.05$ & $0.35 \pm 0.05$ \\
\hline${ }^{2} \Pi_{3 / 2} v=1$ & $7.9 \pm 2.4$ & $3.6 \pm 0.6$ & $0.58 \pm 0.02$ & $0.42 \pm 0.02$ \\
\hline${ }^{2} \Pi_{1 / 2} v=0$ & $-5.3 \pm 2.8$ & $25.7 \pm 2.8$ & $0.55 \pm 0.03$ & $0.45 \pm 0.03$ \\
\hline${ }^{2} \Pi_{1 / 2} v=1$ & $-22.9 \pm 4.2$ & $4.6 \pm 0.8$ & $0.73 \pm 0.04$ & $0.27 \pm 0.04$ \\
\hline${ }^{2} \Pi_{1 / 2} v=2$ & $-19.9 \pm 3.6$ & $3.4 \pm 0.7$ & $0.70 \pm 0.04$ & $0.30 \pm 0.04$ \\
\hline${ }^{2} \Pi_{1 / 2} v=3$ & $-7.1 \pm 3.1$ & $2.9 \pm 0.6$ & $0.57 \pm 0.03$ & $0.43 \pm 0.03$ \\
\hline
\end{tabular}

results and the differences between the $v=0$ and $v=1$ channels at other photon energies confirm that due to the presence of autoionization each channel has to be analyzed separately.

We have presented vibrationally resolved measurements of the partial cross sections $\sigma$ and the polarization parameters $A$ for photoelectrons from HI. Pronounced differences observed for the various final ionic states can be attributed to the presence of strong autoionization structures. The results exemplify the requirement of vibrational resolution for spin-polarization measurements in resonance regions. By partitioning the contributions to the cross section based on the data for $\sigma$ and $A$, the dominance of outgoing $\epsilon l \delta$ waves for the region of electronic autoionization is established quantitatively. This shows that ' $\Pi$ states give the main resonant contribution to the ionization process.

Useful discussions with $H$. Lefebvre-Brion and $G$. Raseev and also help of the BESSY staff is gratefully acknowledged. This work was supported by BMFT (Grant No. 05431 AX/TP2) and the European Commission.
*Present address: Bosch-Siemens Hausgeräte GmbH, D1000 Berlin 20, Germany.

[1] U. Heinzmann, F. Schäfers, and B. A. Hess, Chem. Phys. Lett. 69, 284 (1980); U. Heinzmann, B. Osterheld, F. Schäfers, and G. Schönhense, J. Phys. B 14, L79 (1981).

[2] N. A. Cherepkov, J. Phys. B 14, 2165 (1981).

[3] H. Lefebvre-Brion, A. Giusti-Suzor, and G. Raseev, J. Chem. Phys. 83, 1557 (1985).

[4] G. Raseev, F. Keller, and H. Lefebvre-Brion, Phys. Rev. A 36, 4759 (1987).

[5] G. Raseev and N. A. Cherepkov, Phys. Rev. A 42, 3948 (1990).

[6] M. Büchner, G. Raseev, and N. A. Cherepkov, J. Chem. Phys. (to be published).

[7] T. Huth-Fehre, A. Mank, M. Drescher, N. Böwering, and U. Heinzmann, Phys. Rev. Lett. 64, 396 (1990).

[8] J. L. Dehmer, A. C. Parr, and S. H. Southworth, in Handbook on Synchrotron Radiation, edited by G. V. Marr (Elsevier, New York, 1987), Vol. 2, Chap. 5, p. 241.

[9] T. A. Carlson, Chem. Phys. Lett. 9, 23 (1971).

[10] T. A. Carlson, P. Gerard, M. O. Krause, G. Von Wald, J. W. Taylor, and F. A. Grimm, J. Chem. Phys. 84, 4755 (1986).

[11] A. C. Parr et al., Phys. Rev. A 37, 437 (1988).

[12] N. Böwering, H.-W. Klausing, M. Müller, M. Salzmann, and U. Heinzmann, Chem. Phys. Lett. (to be published).

[13] Ch. Heckenkamp, F. Schäfers, G. Schönhense, and U. Heinzmann, Z. Phys. D 2, 257 (1986).

[14] D. W. Turner, C. Baker, A. D. Baker, and C. R. Brundle, Molecular Photoelectron Spectroscopy (Wiley, New York, 1970), p. 59

[15] T. A. Carlson et al., J. Chem. Phys. 80, 3521 (1984).

[16] N. Böwering, M. Müller, M. Salzmann, and U. Heinzmann, J. Phys. B 24, 4793 (1991).

[17] D. J. Hart and J. W. Hepburn, Chem. Phys. 129, 51 (1989).

[18] A. Mank, M. Drescher, T. Huth-Fehre, N. Böwering, U. Heinzmann, and H. Lefebvre-Brion, J. Chem. Phys. 95, 1676 (1991).

[19] D. T. Terwillinger and A. L. Smith, J. Chem. Phys. 63, 1008 (1975).

[20] P. M. Dehmer and W. A. Chupka, Argonne National Laboratory Report No. ANL-78-65, 1978 (unpublished), Part I, p. 13.

[21] H. Lefebvre-Brion et al., J. Phys. B 22, 3891 (1989).

[22] A. A. Cafolla, J. Comer, and T. Reddish, J. Phys. B 21, 3571 (1988).

[23] H. Lefebvre-Brion and F. Keller, J. Chem. Phys. 90, 7176 (1989). 